\section{$\underset{\substack{\text { hommes } \\ \text { \& migrations }}}{ }$}

\section{Hommes \& migrations}

Revue française de référence sur les dynamiques

migratoires

$1310 \mid 2015$

Fashion Mix

\title{
Étoiles symboliques de la mode noire
}

Chris Seydou, Patrick Kelly et Ozwald Boateng, une perspective comparative

\section{Pascale Berloquin - Chassany}

\section{(2) OpenEdition}

\section{Journals}

Édition électronique

URL : http://journals.openedition.org/hommesmigrations/3157

DOI : 10.4000/hommesmigrations.3157

ISSN : 2262-3353

Éditeur

Musée national de l'histoire de l'immigration

Édition imprimée

Date de publication : 1 avril 2015

Pagination : 77-83

ISBN : 978-2-919040-31-5

ISSN : $1142-852 X$

Référence électronique

Pascale Berloquin - Chassany, "Étoiles symboliques de la mode noire», Hommes \& migrations [En ligne], 1310 | 2015, mis en ligne le 01 avril 2018, consulté le 01 mai 2019. URL : http:// journals.openedition.org/hommesmigrations/3157; DOI : 10.4000/hommesmigrations.3157 


\title{
ÉTOILES SYMBOLIQUES DE LA MODE NOIRE \\ CHRIS SEYDOU, PATRICK KELLY ET OZWALD BOATENG, UNE PERSPECTIVE COMPARATIVE
}

Par PASCALE BERLOQUIN-CHASSANY, docteur en socio-anthropologie, chargée de cours à l'université Paris-XIII Villetaneuse, enseigne la sociologie et l'anthropologie dans des écoles d'infirmières de la région parisienne.

\begin{abstract}
À Paris ou à Londres, l'entrée dans le petit monde feutré des maisons de haute couture n'a pas été chose aisée pour les créateurs noirs. Si la vague ethnique des années 1990 a fortement joué en leur faveur, elle a produit également de nouveaux stéréotypes. Au lieu de s'enfermer dans les attentes que pouvait susciter leur origine supposée, c'est en transgressant les frontières géographiques et culturelles que les créateurs noirs comme Patrick Kelly, Chris Seydou ou Ozwald Boateng sont parvenus à s'imposer dès les années 1980.
\end{abstract}

Il était une fois la haute couture, dont l'ancrage parisien garantissait une renommée internationale. Que la passation de pouvoir en matière des règles du paraitre ait glissé des désirs des monarques français à ceux des créateurs de mode, lentement, tout au long du XIX siècle, mais sûrement, conforte la dimension aristocratique du champ. L'origine britannique de Charles Frederick Worth (18251895), celui par lequel a germé la dynamique des maisons de couture, n'entache nullement son aura parisienne. Parmi les explications, on peut invoquer le soutien de l'impératrice Eugénie et son caractère précurseur dans l'organisation professionnelle du champ. Le luxe resta privilège français car son enjeu est politique. Ce postulat marque les ouvrages consacrés à la mode au XX siècle et minimise mal l'émergence d'autres centres géoculturels (New York depuis la Seconde Guerre mondiale, Londres dans les années 1960, Anvers et Milan depuis les années 1990...).

Le sceau parisien s'applique toutefois, petit à petit, à des couturiers venus d'ailleurs. Du continent européen tel l'Espagnol Cristobal Balenciaga, puis d’Asie avec la reconnaissance des créateurs japonais (Kenzo, Issey Miyake, Comme des Garçons...). En revanche, il n'existe nulle trace de créateurs en provenance du continent africain. La collection Bambara d'Yves Saint Laurent en 1967 indique bien que l'inspiration peut y puiser, mais il s'agit d'une réappropriation française. Plus récemment, 
à la fin des années 1990, le succès de la vague ethnique et du créneau du sportswear ouvre la voie à des personnalités noires. Entre-temps, trois des leurs ont rencontré l'aval parisien. C'est en étudiant la construction iden-

La renommée de Chris

Seydou, dont le prénom est un hommage à Christian Dior repose sur la reconnaissance du champ occidental et l'espoir qu'il a suscité en Afrique. titaire noire au prisme de la création vestimentaire de luxe $e^{1}$ que les noms du Malien Chris Seydou, de l'Africain Américain Patrick Kelly et du Britannique Ozwald Boateng me sont apparus tels des acteurs référents pour leurs confrères actuels. Puisque l'historiographie de la mode, dont le monopole reste encore occidental, tend à les oublier ${ }^{2}$, je vais ici observer l'interprétation noire. L'influence transatlantique de ces trois contes de fées, fées masculines de l'aiguille, se vérifie selon des aléas contextuels en rupture parfois avec certains présupposés.

\section{Du tailleur au couturier : Chris Seydou}

Son histoire est courte : né en 1949 à Kati au Mali, il entre en apprentissage de couture chez des maîtres tailleurs à Ouagadougou au Burkina Faso, puis à Abidjan. En 1971, il suit des cours à l'école Joffrin Byrs à Paris. Huit ans plus tard, il présente son premier défilé dans la capitale française. La renommée de Chris Seydou, dont le prénom est un hommage à Christian Dior ${ }^{3}$ repose sur la reconnaissance du champ occidental et l'espoir qu'il a suscité en Afrique. En 1981, il s'installe à Abidjan et en 1990 à Bamako. Quatre ans plus tard, la Fédération africaine des créateurs du design et de la mode (FAC), dont il est à l'origine, voit le jour au Ghana.
Peu de temps après, le sida l'emporte. Ces quelques informations indiquent très clairement la double stratégie du créateur. Chris Seydou a pris son envol à Paris alors que c'est son attachement à l'Afrique qui ressort principalement de mon corpus. "Il était comme Dieu, pour nous autres", cette citation d'un créateur ivoirien ${ }^{4}$ révèle l'étroite imbrication entre sa personnalité et sa fidélité à ses origines géoculturelles, signifiées par un parcours d'exception ${ }^{5}$. Un de ses amis, lui-même important dans le champ de la mode à Paris, m’a raconté sa générosité hors norme, son humour et son génie créatif. Selon lui, ces qualités ont déterminé son intégration dans le milieu de la couture parisienne et sa familiarité avec les hautes sphères politiques en Afrique. Sa démarche solidaire est d'autant plus remarquable que ses compatriotes ne lui facilitèrent pas la tâche, en témoigne la Togolaise Tanita : “C'est l'innovateur (...). Il a évolué à Abidjan, puis a été foutu à la porte. À la fin de sa vie, il est retourné à Bamako, nul n'est prophète en son pays, lui pas plus qu'un autre. ." Malgré le décalage entre le prestige de son expérience parisienne et la chute douloureuse à son retour sur le continent africain, le voici inscrit au panthéon de la mode africaine.

\section{Une modernisation des tissus traditionnels}

La réussite de Chris Seydou traverse les frontières et sa fin tragique concourt à parfaire sa légende. Des créateurs au Mali, au Togo et en Côte d'Ivoire parlent de lui comme de leur "aîné", "un ami, un frère $e^{7 ”}$. Suggérer des liens personnels avec lui accroît l'aura des créateurs actuels ${ }^{8}$ et les place dans une filiation honorable. Son usage des matériaux dits "traditionnels" a invité certains créateurs à s'auto-

\footnotetext{
1. Cette réflexion prolonge mes recherches de doctorat en socio-anthropologie du monde contemporain, soutenu en décembre 2007 à l'université de Paris-Ouest La Défense-Nanterre : "Reconstruire l'identité noire. La perspective transatlantique des créateurs de mode vestimentaire noirs (France, États-Unis, Caraïbe, Afrique de l'Ouest)." Les noms des créateurs, lorsque leurs propos ne sont pas extraits de la presse, ont été codifiés afin de respecter leur anonymat. 2. Le prisme français qui, jusqu'à peu, traite de manière détournée, sans la nommer, la question raciale, explique le déni relatif de l'altérité noire dans ce champ et l'enjeu de ce type de catégorisation. Cf. Didier Fassin, Éric Fassin (dir), De la question sociale à la question raciale? Représenter la société française, Paris, La Découverte, 2009. 3. Renée Mendy-Ongoundou, Élégances africaines, Paris, Alternatives, 2002, p. 72. 4. Lors du Festival international de la mode africaine (Fima) organisé par le créateur nigérien Alphadi au Niger, 2008. 5. Apprenti tailleur, Chris Seydou quitte l’Afrique pour la France, entre chez Mic Mac, suit son fondateur Tan Guidicelli, puis travaille pour Yves Saint Laurent. 6. Fima, 1998. 7. Ibid.
} 
proclamer ses héritiers spirituels. Selon l'Ivoirien Kokeli, Chris Seydou est, en effet, "le premier à avoir créé de la mode moderne avec des matières africaines". Cette idée s'est largement diffusée depuis ${ }^{10}$. Les dix ans de création de la Camerounaise Ly Dumas, installée à Paris, suscitent un article en hommage à Chris Seydou ${ }^{11}$. La paternité de la rencontre entre le patrimoine africain et la création de luxe lui est attribuée.

Plus qu'esthétique et économique, l'apport du couturier doit être apprécié sous l'angle de la révolution culturelle dont il est l'instigateur ${ }^{12}$. Il est le premier à tailler le bogolan, tissu ancestral et sacré réservé à des rituels comme l'excision. Vider la dimension symbolique des matières pour séduire l'Occident et l'Afrique marque une rupture culturelle considérable. Bruno Badamie, créateur malien installé en Grande-Bretagne, raconte sa surprise "de découvrir un jour dans un magazine un habit en bogolan" et explique alors le rôle fondamental assumé par Chris Seydou: "C'est actuellement un tissu très utilisé par les Dogons. C'est un tissu sacré, qui indique votre position sociale. Or je crois que la première personne à avoir cassé ce cercle était Chris Seydou. Il s'en est servi dans ses modèles, créant ainsi un conflit avec les Dogons, qui ne souhaitaient pas voir leur tissu exploité en Europe ${ }^{13}$." Il a ouvert la voie à d'autres détournements fonctionnels de matériaux traditionnels ${ }^{14}$. Tanita du Togo le souligne : "Avant, il était impensable de découper un pagne, ça représente un dessin global, c'eût été un sacrilège pour grand-maman de couper de tels tissus. En fait, le pagne était plié, respectant le dessin, il se prêtait entre les femmes ${ }^{15}$." Jusque sur le continent africain, la découpe n'est plus un frein à la création. Le texte dédié à Chris Seydou dans le numéro de la Revue Noire sur la mode noire replace sa démarche dans une Afrique postmoderne en devenir, à charge pour ses héritiers de la poursuivre sans s'enferrer dans la tradition ${ }^{16}$. À valoriser les matériaux et les motifs africains au rythme des coupes européennes ${ }^{17}$, le couturier aurait été "agacé par LE style africain qu'il avait lui-même forgé, il voulait se démarquer de la société actuelle africaine dans ses contradictions entre l'identité étroite liée au patrimoine et la modernité urbaine banalisée qu'il aimait vivre". L'influence européenne devait être intégrée "pour porter la mode africaine à maturité18”. Cette vision autocritique qui lui est attribuée ne paraît nullement dans la presse féminine noire. Le "tailleur", Chris Seydou, en accédant au statut de "couturier" par son expérience parisienne, démontre que l'apprentissage en Afrique peut mener loin, jusqu'à l'entrée dans un champ occidental peu enclin à l'altérité noire. Sa réussite réside également dans la fierté africaine qu'il a su mettre en avant. Celle de Patrick Kelly, Noir américain, est moins perceptible.

\section{Patrick Kelly, un Américain à Paris}

Dans la lignée de son idole Joséphine Baker, Patrick Kelly (1954-1990), originaire du Mississipi aux États-Unis et formé à la Parsons School of Design à New York, se rend à Paris en 1979 pour tenter sa chance. Il vend ses vêtements couverts de boutons colorés dans la rue, puis il présente sa première collection en 1985. Il est le premier Américain à intégrer la Chambre syndicale du prêt-àporter des couturiers et des créateurs de mode en 1988. De plus, dans les rares ouvrages occidentaux sur la mode qui le mentionnent, sa couleur de peau ne l'est pas ${ }^{19}$. L'explication peut provenir

8. Il a encouragé Gilles Touré, alors âgé de 12 ans, en réalisant un de ses croquis et conseillé la Gabonaise Olga'O. Cf. Divas, février-mars 20oo, pp. 62-63, et Divas, avril-mai 2000, pp. 64-65. Voir également la dynamique impulsée par Mariah Bocoum Keita avec le Chris Seydou Fashion Show en 2011 à Bamako, puis ses nombreuses mentions du couturier pour arguer que "les héritiers de Chris Seydou rallument le flambeau" (http://maliactu.net/promotion-de-la-mode-au-mali, 15 janvier 2014). 9. Fima, 1998. 10. Renée Mendy-Ongoundou, op. cit., p. 71. 11. Leur rencontre a déclenché sa vocation : "Cet homme d'exception [qui] m'a impressionnée par ses collections de bogolan si bien travaillé" (Amina, mai 2001, p. 10). 12. Au point de voir sa créativité qualifiée a posteriori de "forme de résistance à la mondialisation" dans un blog dédié à la culture malienne : "Chris Seydou, l'homme qui valorisa dans le monde entier la mode malienne". http://cultures-maliennes.overblog.com, 20 septembre 2012. 13. Amina, novembre 1999, p. 33. 14. Anne Grosfilley, Afrique des textiles, Aix-en-Provence, Edisud, 2004, p. 94 15. Fima, 1998. 16. Revue Noire, décembre-janvier 1998-1999, p. 5. 17. Comme le relate la revue Amina, par exemple, en recensant des "tailleurs à la 'Chanel', boubous sophistiqués en passant par les robes manteaux où le pagne retrouve ici toute sa dignité" (Amina, juillet 1980, p. 12). 18. Ibid., p. 7 . 
de la tolérance accordée aux artistes noirs à Paris depuis les années $1920^{20}$. Que penser, en revanche, du silence relatif de la presse féminine noire américaine interrogée à son égard ? Est-ce en raison d'une vision spécifique de l'identité noire que son succès n'a pas été médiatisé pour servir d'exemple à ses confrères outre-Atlantique? Aux États-Unis, où le particularisme est vif, Patrick Kelly devient tardivement un symbole.

Avant son décès dû au sida, le $1^{\text {er }}$ janvier 1990 , le nom de Patrick Kelly associé à une appartenance noire paraît rarement dans

Lappartenance noire Jet ou Ebony ${ }^{21}$. L'examen d'Esde Patrick Kelly apparaît bien en fonction des contextes d'énonciation. À ses débuts,

les discours de la presse

féminine noire américaine

étaient manifestement peu enclins à valoriser son étiquette noire. sence Magazine révèle la primauté occidentale de sa légitimité. En 1989, la revue titrait "Patrick Kelly : Prince de Paris. Créateur de mode américain à Paris" et aucune caractéristique noire n'était sensible à moins de déchiffrer consciencieusement l'article ${ }^{22}$. Ce n'est qu'après sa disparition qu'il est progressivement érigé en icône de la mode noire. En 1991, un texte signale des "Frères sur la $7^{e}$ avenue" en prenant appui sur l'exemple Patrick Kelly qui a "dû devenir une star à Paris avant d'être reconnu par l'industrie de la mode américaine". S'il incarne a posteriori une figure valorisante et récente dans l'histoire de ces créateurs, l'essentiel du développement concerne d'autres parcours ${ }^{23}$. En 1994, Essence Magazine inscrit un autre créateur noir célèbre à Paris, Xuly Bët, dans la même "tradition" que Patrick Kelly, à savoir "spunky, funky". Le style streetwear les réunirait au même titre que l'idée de récupération ${ }^{24}$. Au-delà de sa fantaisie, c'est surtout sa touche française qui est valorisée. Le créateur noir Patrick Robinson le désigne comme "la crème de la création française 25 ". Dans l'ouvrage de Constance C.R. White, Style Noir, le titre du chapitre dédié à la contribution des Noirs dans la mode commence par : "From Kente to Kelly $(\text {... })^{26}$."Il faut pourtant lire 45 pages avant d'accéder à une brève description de ce créateur. Seule sa marque de fabrique - des pin's de poupées noires - peut signaler une spécificité noire. L'exubérance du personnage et sa créativité ont pourtant marqué ses confrères en France ${ }^{27}$. Ainsi, Piola du Sénégal m'en parla tel un ami de longue date : "Lui, c'était un Noir américain très équilibré, je veux dire il avait pas de faux problèmes ${ }^{28}$." Que signifiaient les expressions "très équilibré" et "faux problèmes" ? Recherchait-il ses racines africaines ? Piola écarta cette idée : "Non, il vivait son truc, et puis c'est tout." Et son "truc" n'était peut-être pas l'identité noire. Patrick Kelly a donc marqué des créateurs noirs parmi d'autres, mais pas spécifiquement en raison de l'expression d'un style noir. Cette hypothèse se modifie pourtant à l'épreuve du temps. Au début des années 2000, l'ambivalence de Patrick Kelly paraît sous un jour nouveau, très noire finalement.

Sur le site Internet nord-américain des créateurs de mode noirs, j'ai fini par trouver la cause du malaise de ses contemporains noirs. L'ostentation de petites poupées noires ${ }^{29}$ prête à la controverse. En dépit d'une clientèle célèbre, l'image infantile et sa créativité urbaine "oversize overalls ${ }^{30 "}$ ne répondaient guère aux attentes des Africains américains. Le caractère provocateur de ces pin's étaitil trop précoce ? Parallèlement, le rap connaissait un succès certain. Patrick Kelly appelait à la prise de conscience de son passé d'opprimé pour l'assumer et ne saisissait pas le caractère dérangeant de

\footnotetext{
19. Boucher dans son Histoire du costume le cite parmi la troisième vague de créateurs en vogue à Paris au milieu des années 1980. Cf. François Boucher, Histoire du costume, Paris, Flammarion, 1996, p. 431. Laurence Benaïm repère, pour sa part, son originalité dans ses robes de mariée. Cf. Benaïm Laurence, L'Année de la mode, 1988-1989, Paris, La Manufacture, 1990, pp. 101-103. 20. Pascal Blanchard, Gilles Manceron, Éric Deroo, Le Paris noir, Paris, Hazan, 2001. 21. Ebony, qui lui consacre un article en février 1988 (p. 52 ), le signale en octobre suivant également comme un créateur noir (p. 114), contrairement à Jet, qui le cite parmi les grands noms de couturiers sans le préciser noir. A posteriori, le lecteur de Jet peut faire cette association grâce à la photographie qui illustre l'article rendant hommage au créateur (Jet, 22 janvier 1990, pp. 24 et 26). 22. Essence Magazine, mai 1989. 23. Ibid., novembre 1991, pp. 73-74. 24. Le créateur américain utilisait des boutons colorés comme sa grand-mère, qui dissimulait ainsi l'usure de ses vêtements (ibid., mai 1994, p. 26). 25. Ibid., septembre 1995, pp. 22 et 128. 26. Constance C.R. White, Style Noir. The First How-to Guide to Fashion Written with Black Women in Mind, New York, A Perigee Book, 1998, pp. 54-56. 27. Notons que Patrick Kelly ne figure pas dans le numéro de la Revue Noire sur la mode (décembre-janvier 1998-1999, p. 5) ni dans l'ouvrage de René Mendy, dans lequel Mike Sylla - Baïfall Dream - lui rend néanmoins hommage. Cf. Renée Mendy-Ongoundou, op. cit., p. 100. 28. Fima, 2000. 29. Pour compenser la frustration de son enfance où elles n'existaient pas. 30. Salopette trop grande.
} 


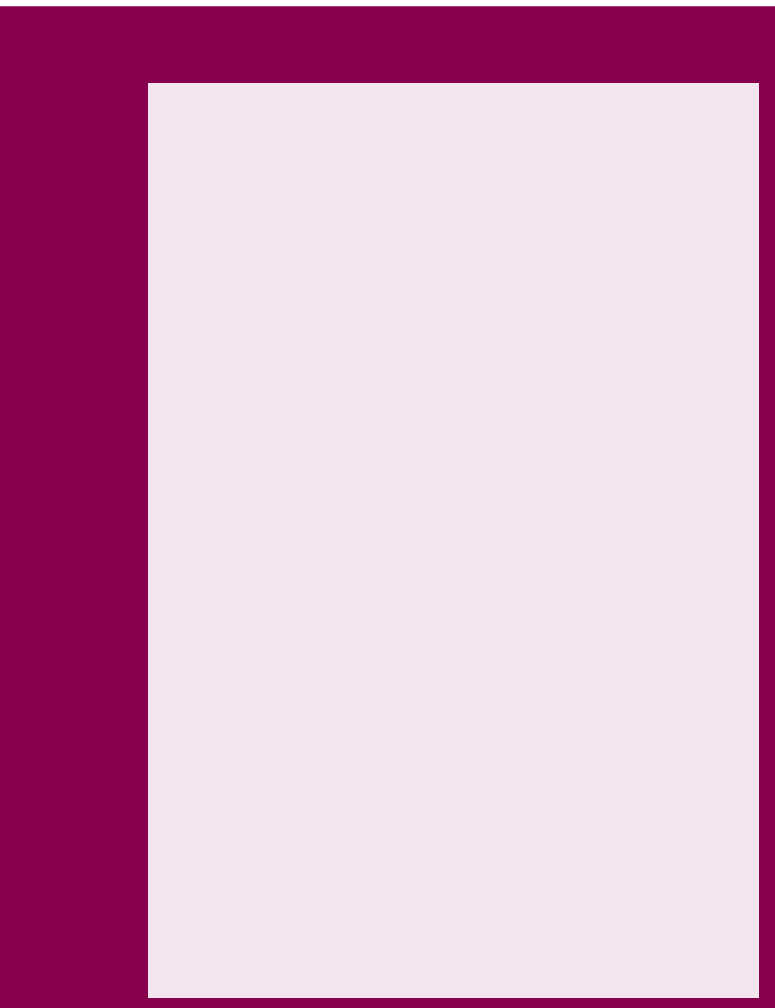

L'Afrique de la mode au Palais de la Porte Dorée, novembre 2010 (c) Abdelkader Benamer / D. Langoutte

ses poupées ${ }^{31}$. Un ouvrage occidental interprète, au contraire, cette dynamique comme fédératrice ${ }^{32}$. L'appartenance noire de Patrick Kelly apparaît bien en fonction des contextes d'énonciation. À ses débuts, les discours de la presse féminine noire américaine étaient manifestement peu enclins à valoriser son étiquette noire. Progressivement, et jusqu'en 2014 avec l'exposition de son travail au Musée des arts de Philadelphie, son nom nourrit une filiation spécifique avec la mode noire. C'est principalement son style qui mobilise dès lors l'attention des journalistes. Son talent humoristique à manier les stéréotypes est associé à un besoin de les dépasser, au point d'avoir été perçu comme Américain avant de l'être comme Noir par ses confrères nord-américains. Les tiraillements identitaires noirs empruntent une tournure inattendue dans le cas d'Ozwald Boateng.

\section{Du tailleur au directeur artistique : Ozwald Boateng}

Autodidacte, il s'installe dès l'âge de 27 ans en 1995 à Londres dans le quartier de Savile Row, célèbre pour son regroupement de tailleurs traditionnels bespoke. Ce terme renvoie à une pratique du sur-mesure fidèle au moindre désir du client ${ }^{33}$. Sa renommée ${ }^{34}$ séduit en 2003 Givenchy, qui lui confie la direction artistique de sa branche "Homme" et devient donc l'expression d'une marque de couture française ${ }^{35}$. Dans quelle mesure ses origines ghanéennes s'articulent-elles à un discours identitaire noir?

Dans un article publié le 24 janvier 1995 dans Libération, la prédilection d'Ozwald Boateng pour des couleurs vives est rattachée à son origine africaine. Mais, concrètement, peu de journalistes s'y intéressent ${ }^{36}$. L'important réside dans son style. Célèbre pour son élégance et son originalité, Ozwald Boateng excelle dans un domaine traditionnel, ce qui suscite le trouble. La tradition à laquelle il est fait référence est bien britannique

31. II confie ainsi à Essence Magazine en mai 1989: "Récemment, quelqu'un de Noir m'a dit qu'ils étaient stressés à l'idée de porter les poupées noires miniatures en pin's. Et j'ai pensé, tu peux porter un pistolet ou un équipement guerrier de camouflage et les gens trouvent ça tellement chic, mais si tu portes un petit bébé noir en pin's, les gens t'attaquent. Je fais ces choses pour que nous ne nous oublions pas." 32 . L'article qui lui est consacré dans le livre du Musée de la mode retient sa "fantaisie". Les boutons et les "poupées de chiffon noir, jadis signes d'oppression" illustrent la gaieté contagieuse. L’appartenance noire est évasive jusqu'à la conclusion: “De 1985 à 1989, la mode jeune et endiablée de Kelly met en scène la jeunesse et la communauté noires." (Paris, Phaidon, 2004, p. 244). 33. http://www.eurweb.com/story, juin 2006. 34. James Laver le présente comme l'un des Britanniques qui a révolutionné le métier de tailleur. Cf. James Laver, Histoire de la mode et du costume, Paris, Thames \& Hudon, 2003, p. 285. 35. II reste dans cette maison jusqu'en 2007. Il est depuis surnommé "the Cut Killer" (le Tueur de la coupe) pour avoir rajeuni l'image de Givenchy (Le Point, 9 décembre 2014). 36. Son lieu de naissance varie selon l'appartenance identitaire de l'énonciateur : à Londres pour l'encyclopédie Wikipédia, il est “issu d'une famille ashantie du Ghana”; le site consacré au luxe ne se prononce pas ("Givenchy, la divine surprise”, 2005). Sa composante britannique est privilégiée : il est reconnu parmi les 100 Great Black Britons en 2004 et parmi ceux de l'Ordre de l’Empire britannique en 2006. 37. Revue Noire, décembre-janvier 1998-1999, p. 65. 
et concerne la réalisation de costumes. Même si la Revue Noire lui prête une dynamique revitalisatrice - "par son panache d'enfant terrible du dandysme contemporain $^{37 "}$-, l'esprit est bien britannique ${ }^{38}$. Certes, un article - "Ozwald

La presse féminine noire témoigne de la prégnance des référents africains chez Chris Seydou, de leur invisibilité chez Ozwald Boateng et du caractère tardif de la dimension noire reconnue à Patrick Kelly.

Boateng. L'Africain que tout le monde veut" - souligne un lien avec le Ghana en concluant : "Malgré cette réussite, il n’oublie pas ses origines. En 1990, il foule pour la première fois le sol ghanéen. Il a même été décoré par le chef de la tribu ashanti, à laquelle appartient sa famille.

Pour son pays, il a des projets et compte les rendre effectifs bientôt $t^{39}$." L'omniprésence des références à cette image du couturier bespoke, avec cette élégance et ce style britanniques faisant office de modèle identitaire, explique qu'une interprétation autre de son image soit hésitante. Ozwald Boateng investit la haute couture et son exemple doit encourager les autres créateurs noirs à poursuivre leurs efforts ${ }^{40}$. Depuis 2007, son intérêt pour le continent noir se concrétise par l'orchestration du $9^{e}$ sommet de l'Union africaine au Ghana, puis avec la naissance de sa fondation Made in Africa en 2011. La réalisation d'un documentaire, A Man's Story, souligne plus encore son appartenance noire en relatant le parcours d'un couturier noir dans Savile Row ${ }^{41}$.

\section{Transgresser les codes}

Quand Chris Seydou a tenté d'exploiter son succès parisien pour le partager avec ses pairs, à son retour en Côte d'Ivoire puis au Mali, celui de Patrick Kelly aux États-Unis procède des médias noirs. Ces derniers réinterprètent son impact identitaire à l'aune de son appartenance noire, mais son corps demeure à Paris. Ozwald Boateng opère bien des allers-retours à ses origines symboliques, comme si sa notoriété pouvait nourrir celle du continent africain. Mais son pays reste, pour l'instant, la GrandeBretagne. Chris Seydou, Patrick Kelly et Ozwald Boateng symbolisent la possibilité d'atteindre le sommet lorsque l'on est créateur et noir. L'élaboration des discours identitaires n'est pas linéaire car elle dépend du créateur et du contexte. La presse féminine noire témoigne de la prégnance des référents africains chez Chris Seydou, de leur invisibilité chez Ozwald Boateng et du caractère tardif de la dimension noire reconnue à Patrick Kelly. En 1998, la mémoire de Chris Seydou était suffisamment vive pour apparaittre dans mes entretiens. Au fil du temps, ceux qui s'y réfèrent souhaitent être associés à une lignée honorable. L'exposition des créations de Patrick Kelly au Musée de Brooklyn est organisée quatorze ans après sa mort, en 2004. Un tel délai correspond à celui de la banalisation du phénomène hip-hop et de l'instrumentalisation des stéréotypes noirs qui en découle. Avec ses poupées politiquement incorrectes en leur temps pour la communauté noire nord-américaine, Patrick Kelly est, à mon sens, précurseur d'une relation provocatrice avec le passé noir. Dans les trois cas, "la politique d'accomplissement ${ }^{42}$ ", ou stratégie d'adaptation/intégration, est la plus visible, voire la plus valorisée. Ce n'est qu'avec Chris Seydou que cet atout nourrit une réappropriation africaine, c'est-à-dire une "politique de transfiguration ${ }^{43}$ ". Si un style fantaisiste rassemble les trois créateurs, seuls Patrick Kelly et Chris Seydou ont manifesté, certes diversement, leur appartenance noire. Ces différentes réappropriations à visée identitaire de trois étoiles de la mode noire ne concernent pas directement leur réception dans la société française. Pour autant, leurs succès indiquent en filigrane trois tendances chères à d'autres créateurs noirs, mais pas seulement.

Le travail de Chris Seydou, à l'aube des années 1980, présente des similitudes avec la vague qualifiée d'ethnique quinze ans plus tard. Il découpe pagnes et bogolans selon des préceptes occiden-

38. Je le vérifie dans des articles dont les titres prédisent un discours particulariste : finalement, aucune autre allusion au continent africain n’y figure (Miss Ébène, juillet-août, 2001, p. 39 ; Divas, février, 2004, p. 17). 39. http://www.topvisages.net. 
taux. C'est-à-dire qu'il porte sur des podiums parisiens la rencontre entre des éléments dits "de tradition africaine" et d'autres perçus alors comme ceux de la "modernité" (coupes tailleurs, épaulettes, jupes courtes...). Représentant de ses confrères et conscurs ouest-africains, il leur a montré la voie d'une réussite possible à Paris, qu'il souhaitait également et de manière collective sur son continent d'origine. Son empreinte symbolique mêle ainsi sa créativité et un désir d'unir celle d'acteurs africains pour refaçonner la mode internationale.

La comète Patrick Kelly pourrait sembler plus individualiste au regard des interprétations noires nord-américaines si l'on omettait celles qui fleurissent depuis l'exposition qui lui a été consacrée en 2014 par le Musée des arts de Philadelphie. Son parcours parisien fulgurant illustre l'amour des autres quel qu'il soit ${ }^{44}$, mais aussi son héritage noir qu'il s'agirait d'exorciser à l'instar du petit golliwog ${ }^{45}$ sculpté sur sa tombe après avoir orné ses sacs publicitaires. L'humour et la gaieté traduits par l'usage de boutons colorés, la robe banane portée par Pat Cleveland en clin d'œil à Joséphine Baker, servent non seulement à jouer des stéréotypes ${ }^{46}$, mais à proposer une mode urbaine, longiligne, minimaliste, dans la lignée du streetwear de luxe ${ }^{47}$, dont l'origine nord-américaine ne cesse de rayonner.

Le parcours d'Ozwald Boateng, que certains peinent à rattacher au continent africain autrement que par son origine familiale, ouvre concrètement une voie classique dans la haute couture. La mâ̂trise des codes de Savile Row qu'il a su concilier avec la virtuosité de la couture française en travaillant pour Givenchy atteste l'existence d'une solution en dehors d'une inscription dans un particularisme africain. Non pas qu'il faille s'éloigner de ses origines, et son investissement dans une fondation, Made in Africa, le prouve, mais la filiation culturelle première de sa créativité peut être étiquetée autre : britannique dans son cas.

À travers l'expression, et surtout l'interprétation qui en est faite, ces trois créateurs de luxe parisien offrent un panel d'espoirs quant à l'expression noire, qu'elle soit qualifiée d'ethnique, d'urbaine (streetwear) ou de classique dans le sens occidental du terme ${ }^{48}$. Force est de constater l'amplitude du champ des possibles signalée par ces trois étoiles parmi d'autres qui ne demandent qu'à briller. Le point commun qui ressort de cet examen est la transgression des frontières, de ces stéréotypes associés à un phénotype ou à des origines culturelles. Les progrès en matière de communication amplifient la circulation des idées au point qu'en Afrique des informations sont disponibles sur Patrick Kelly, et sur Ozwald Boateng. Il en est de même aux États-Unis, où le nom de Chris Seydou les accompagne. Si la référence est parisienne, le rayonnement de ces créateurs est international, dépasse les différences culturelles, voire raciales. Pour preuve, l'expression du désir de poursuivre l'héritage de Patrick Kelly ou celui de Chris Seydou par, respectivement, Gerlan Marcel (Gerlan Jeans) à New York et Mariah Bocoum Keita (Les Péchés Mignons) à Bamako. 